TRANSACTIONS OF THE

AMERICAN MATHEMATICAL SOCIETY

Volume 349, Number 11, November 1997, Pages 4343-4353

S $0002-9947(97) 01529-8$

\title{
ESSENTIAL EMBEDDING OF CYCLIC MODULES IN PROJECTIVES
}

\author{
JOSÉ L. GÓMEZ PARDO AND PEDRO A. GUIL ASENSIO
}

\begin{abstract}
Let $R$ be a ring and $E=E\left(R_{R}\right)$ its injective envelope. We show that if every simple right $R$-module embeds in $R_{R}$ and every cyclic submodule of $E_{R}$ is essentially embeddable in a projective module, then $R_{R}$ has finite essential socle. As a consequence, we prove that if each finitely generated right $R$-module is essentially embeddable in a projective module, then $R$ is a quasiFrobenius ring. We also obtain several other applications and, among them: a) we answer affirmatively a question of Al-Huzali, Jain, and López-Permouth, by showing that a right CEP ring (i.e., a ring $R$ such that every cyclic right module is essentially embeddable in a projective module) is always right artinian; b) we prove that if $R$ is right FGF (i.e., any finitely generated right $R$-module embeds in a free module) and right CS, then $R$ is quasi-Frobenius.
\end{abstract}

\section{INTRODUCTION}

It is well known that a ring $R$ is quasi-Frobenius $(\mathrm{QF})$ precisely when every right $R$-module embeds in a projective or, equivalently, in a free module. More generally, $R$ is called right FGF when every finitely generated right $R$-module embeds in a free module. It is also known that if $R$ is left and right FGF (or, more generally, if every cyclic left and right module embeds in a free module), then $R$ is, again, a QF ring, but it is still an open problem whether a right FGF ring is QF. This problem appeared first in Levy's paper [12] as a question for right Ore rings, and it was later formulated in the present form by Faith (see [6] for a thorough discussion of the problem, and also [7] for a more recent update). There is a long list of conditions that force a right FGF ring to be QF, among them, that of $R_{R}$ having finite essential socle.

The most powerful result among those providing this condition is Osofsky's theorem [14] that asserts that a right injective cogenerator ring (i.e., a right $\mathrm{PF}$ ring) is semiperfect and has finite essential right socle. This was the basis of the proof by Björk [2] that right FGF right self-injective rings are QF, a result also obtained, independently, by T.S. Tolskaya (cf. [6]). More generally, Menal [13] used a modification of Osofsky's arguments to prove that if each cyclic right $R$-module embeds in a free module and the injective envelope $E\left(R_{R}\right)$ is projective, then $R$ is $\mathrm{QF}$.

Received by the editors December 2, 1994 and, in revised form, May 2, 1995.

1991 Mathematics Subject Classification. Primary 16L60, 16L30; Secondary 16D50, 16E50, $16 \mathrm{~S} 50$

Work partially supported by the DGICYT (PB93-0515, Spain). The first author was also partially supported by the European Community (Contract CHRX-CT93-0091) and the Xunta de Galicia (XUGA 10502B94).

(C)1997 American Mathematical Society 
Here, we will also use Osofsky's proof as a starting point to develop a technique that allows us to deal with rings such that (certain) cyclic or finitely generated modules are essentially embeddable in projective modules. Our idea is to trade off the additional conditions required to prove that a right FGF ring is QF for a tighter embedding hypothesis. This allows us to approach the Levy-Faith problem from a new perspective, and to give a positive answer for it in an interesting case, by showing that if every finitely generated right $R$-module is essentially embeddable in a projective, then $R$ is QF. We feel that our proof provides some insight on the difficulties involved in obtaining a positive solution to the general FGF problem, and adds support to the conjecture (see [13]) that right FGF rings are not necessarily QF. Things are pretty close, however, and our methods allow us to prove another positive result that also extends the Tolskaya-Björk theorem: a right FGF right CS ring is QF.

Another motivation for the study of these essential embeddings comes from the structure results on rings $R$ such that all cyclic right $R$-modules are essentially embeddable in projectives (right CEP rings) and rings $R$ such that the cyclic right $R$-modules are essentially embeddable in direct summands of $R_{R}$ (right CES rings), obtained in $[1,10,9]$. Our results allow us to answer affirmatively the question posed in [1] of whether a right CEP ring (and, consequently, a right CES ring) is already semiperfect. Among the consequences we mention that the structure theorem for semiperfect right CES rings obtained in [9] becomes, in fact, a structure theorem for the class of all right CES rings.

These results arise as a consequence of our main theorem (Theorem 3.1), in which we prove, in a slightly more general form, that if $R$ is a ring such that $E=E\left(R_{R}\right)$ is a cogenerator and every cyclic submodule of $E_{R}$ is essentially embeddable in a projective module, then $R_{R}$ has finite essential socle. This extends both Osofsky's and Menal's results mentioned above. The method of proof involves, as main ingredients, an adaptation of Osofsky's counting argument applied to certain families of simple right $S / J$-modules (where $S=\operatorname{End}\left(E_{R}\right)$ and $J=J(S)$ is the Jacobson radical of $S$ ), and the construction of an injective mapping from the set of isomorphism classes of one of these families to the set of isomorphism classes of simple right $R$-modules. It is, precisely, in the construction of this injection that the essential embedding hypothesis plays a fundamental role.

Throughout this paper, all rings $R$ will be associative and with identity, and Mod- $R$ will denote the category of right $R$-modules. By a module we will usually mean a right $R$-module and, whenever we want to emphasize the fact that $M$ is a right $R$-module, we will write $M_{R}$. We refer to [5] for all undefined notions used in the text.

\section{Preparatory lemmas}

Let $R$ be a ring, $E_{R}$ an injective module, $S=\operatorname{End}\left(E_{R}\right)$, and $J=J(S)$. Then $S / J$ is a regular right self-injective ring, and idempotents lift modulo $J$. Our first step will be to make precise the connection between indecomposable direct summands of $E_{R}$ and minimal right ideals of $S / J$.

Lemma 2.1. Let $R$ be a ring, $E_{R}$ an injective module, $S=\operatorname{End}_{R}(E)$, and $J=$ $J(S)$. Then there exists a bijective mapping from the set of isomorphism classes of indecomposable direct summands of $E_{R}$ to the set of isomorphism classes of minimal right ideals of $S / J$. 
Proof. Let $L_{R}$ be a direct summand of $E_{R}$. Then we have a canonical isomorphism $\operatorname{Hom}_{R}(E, L) \otimes_{S} E \cong L$. Similarly, if $N_{S}$ is a direct summand of $S_{S}$, then $\operatorname{Hom}_{R}\left(E, N \otimes_{S} E\right) \cong N$. Thus, the assignments $L \mapsto \operatorname{Hom}_{R}(E, L)$ and $N \mapsto N \otimes_{S} E$ define inverse bijections between the sets of isomorphism classes of direct summands of $E_{R}$ and $S_{S}$. It is also clear that these bijections preserve the property of being indecomposable. Actually, if $e \in S$ is the idempotent associated to the direct summand $L$ of $E$ (so that $L=e E \cong e S \otimes_{S} E$ ), then the corresponding direct summand of $S_{S}$ is precisely $\operatorname{Hom}_{R}(E, e E) \cong e S$. Since $\operatorname{End}\left(e E_{R}\right) \cong e S e \cong \operatorname{End}\left(e S_{S}\right)$, we have that if $e E_{R}$ is indecomposable, then the corresponding direct summand $e S$ of $S_{S}$ is an indecomposable projective module with local endomorphism ring. It is well known that $e S e$ is local if and only if $e S / e J$ is a simple right $S$-module whose projective cover is precisely $e S$. But $e S / e J$ is isomorphic, as right $S / J$-module, to the minimal right ideal $\bar{e}(S / J)$ of $S / J$, where $\bar{e}=e+J$, and so we may assign to $e S$ this minimal right ideal.

Conversely, since $S / J$ is a von Neumann regular ring and idempotents lift modulo $J$, each minimal right ideal of $S / J$ is of the form $\bar{e}(S / J)$, where $e \in S$ is an idempotent and $\bar{e}=e+J$. Then we can assign to $\bar{e}(S / J)$ the right ideal $e S$ of $S$, which is, clearly, a projective cover in Mod- $S$ of $\bar{e}(S / J)$, so that $e S e$ is local. Hence, we get a bijection between isomorphism classes of direct summands of $S_{S}$ with local endomorphism rings and isomorphism classes of minimal right ideals of $S / J$, which completes the proof.

Our next step will be to adapt Osofsky's counting argument in [14] to our situation. In order to state it more clearly, we need the following definition.

Definition 2.2. Let $S$ be a ring. A family $\left\{C_{k}\right\}_{k \in K}$ of simple right $S$-modules will be called an idempotent-orthogonal family of simple modules when they are pairwise nonisomorphic and there exists a family $\left\{e_{k}\right\}_{k \in K}$ of idempotents of $S$ satisfying $C_{j} e_{k}=0$ if $j \neq k$, and $C_{k} e_{k} \neq 0$ for each $k \in K$.

The next lemma embodies Osofsky's counting argument, and its proof is essentially contained in [14, Lemma 8 , Theorem 1$]$. We denote by $|X|$ the cardinality of a set $X$.

Lemma 2.3. Let $Q$ be a regular right self-injective ring, and let $\left\{C_{i}\right\}_{i \in I}$ be a set of pairwise nonisomorphic minimal right ideals of $Q$. If $I$ is an infinite set, then there is an idempotent-orthogonal family $\left\{C_{k}\right\}_{k \in K}$ of simple right $Q$-modules such that $|I|<|K|$.

Proof. For each subset $J \subseteq I$, define $\Sigma(J):=\Sigma\left\{C \subseteq Q_{Q} \mid C \cong C_{i}\right.$ for some $\left.i \in J\right\}$, and let $E(\Sigma(J))$ be its injective envelope. Since $Q$ is right self-injective, $E(\Sigma(J))$ embeds in $Q_{Q}$ and so there exists an idempotent $e_{J} \in Q$ such that $E(\Sigma(J)) \cong e_{J} Q$. Following the proof of [14, Lemma 8], we first show that $e_{J}$ is central. It is enough to prove that, for every $x \in Q, e_{J} x=e_{J} x e_{J}$ and $x e_{J}=e_{J} x e_{J}$. Assume, then, that $e_{J} x\left(1-e_{J}\right) \neq 0$. Since $\Sigma(J)$ is essential in $e_{J} Q$, we see that there exist an element $q \in Q$ and a simple right $R$-module $C$ such that $C \cong C_{i}$ for some $i \in J$ and $0 \neq e_{J} x\left(1-e_{J}\right) q \in C$. Thus the homomorphism $\left(1-e_{J}\right) q Q \rightarrow C$ given by left multiplication with $e_{J} x$ is nonzero and, since $C_{Q}$ is a projective module, it is actually a split epimorphism. Therefore, $\left(1-e_{J}\right) Q$ contains a simple submodule isomorphic to $C_{i}$, for some $i \in J$, which is in contradiction with the fact that all these submodules are contained in $e_{J} Q$. Thus we have proved that $e_{J} Q\left(1-e_{J}\right)=0$. 
Assume now that $\left(1-e_{J}\right) x e_{J} \neq 0$ for some $x \in Q$. Then, left multiplication with $\left(1-e_{J}\right) x$ gives a nonzero homomorphism $e_{J} Q \rightarrow\left(1-e_{J}\right) Q$. Its image $\left(1-e_{J}\right) x e_{J} Q$ is a principal right ideal of $Q$ contained in $\left(1-e_{J}\right) Q$, and, since $Q$ is regular, it is a projective module. Hence $e_{J} Q$ contains a submodule isomorphic to $\left(1-e_{J}\right) x e_{J} Q$. But the latter module has zero intersection with $e_{J} Q$ and thus contains no submodule isomorphic to $C_{i}$, for any $i \in J$, which contradicts the fact that $\Sigma(J)$ is essential in $e_{J} Q$. Thus we see that all the $e_{J}$ are indeed central idempotents.

Next, we construct the idempotent-orthogonal family $\left\{C_{k}\right\}_{k \in K}$. As in the proof of [14, Theorem 1], the infinite set $I$ can be decomposed, using a lemma due to Tarski, as the union of a class $K$ of subsets of $I$ such that the following conditions are satisfied:

(i) $|K|>|I|$,

(ii) $|X|=|Y|$ for each $X, Y \in K$,

(iii) $|X \cap Y|<|X|=|Y|$ if $X \neq Y(X, Y \in K)$.

For each subset $X$ of $I$ we have $\Sigma(X)=\Sigma(X \backslash Y) \oplus \Sigma(X \cap Y)$, so that $E(\Sigma(X))=$ $E(\Sigma(X \backslash Y)) \oplus E(\Sigma(X \cap Y))$, and hence $e_{X} Q=e_{X \backslash Y} Q \oplus e_{X \cap Y} Q$. Thus we see that $e_{X \cap Y} \in e_{X} Q$ and, similarly, $e_{X \cap Y} \in e_{Y} Q$. Consequently, $e_{X \cap Y} \in e_{X} Q \cap e_{Y} Q \subseteq$ $e_{X} e_{Y} Q=e_{Y} e_{X} Q$. On the other hand, if $C$ is a simple right $Q$-module contained in $e_{X} e_{Y} Q$, then $C \cong C_{i}$ for some $i \in X$ and, similarly, $C \cong C_{j}$ for some $j \in Y$. Thus $C_{i} \cong C_{j}$ and so $i=j \in X \cap Y$. Therefore, $C \in \Sigma(X \cap Y)$. Since $e_{X} e_{Y} Q$ has essential socle, we have that $e_{X} e_{Y} Q \subseteq E(\Sigma(X \cap Y))=e_{X \cap Y} Q$, and so $e_{X \cap Y} Q=e_{X} e_{Y} Q$. Thus we see that $e_{X} e_{Y} Q$ is a ring in which both $e_{X} e_{Y}$ and $e_{X \cap Y}$ are identities, and hence $e_{X} e_{Y}=e_{X \cap Y}$.

Now let $N=\Sigma\left\{e_{Z} Q \mid Z \subseteq I\right.$ and $|Z|<|X|$ for each $\left.X \in K\right\}$ and define, for each $X \in K, N_{X}=\left(1-e_{X}\right) Q+N$. Since $e_{X}$ and all the $e_{Z}$ are central idempotents of $Q$, both $N$ and $N_{X}$ are two-sided ideals of $Q$. Assume that $e_{X} \in N_{X}$. Then we may write $e_{X}=\left(1-e_{X}\right) x_{0}+n$, with $x_{0} \in Q, n \in N$. Thus there exist sets $Z_{1}, \ldots, Z_{r} \subseteq I$ such that $\left|Z_{j}\right|<|X|$ for $j=1, \ldots, r$, and $n=\sum_{j=1}^{r} e_{Z_{j}} x_{j}$, with $x_{j} \in Q$. Since $|X|$ is infinite, we have that $\left|\bigcup_{j=1}^{r} Z_{j}\right|<|X|$, and hence there exists an element $i \in X \backslash\left(\bigcup_{j=1}^{r} Z_{j}\right)$. But, since $e_{X} Q=\left(1-e_{X}\right) x_{0} Q+\sum_{j=1}^{r} e_{Z_{j}} x_{j} Q$, we then have that $e_{X} Q$ does not contain any simple submodule isomorphic to $C_{i}$. This contradicts the fact that $i \in X$ and shows that $e_{X} \notin N_{X}$. On the other hand, if $X, Y \in K$ and $X \neq Y$, then $e_{Y}=\left(1-e_{X}\right) e_{Y}+e_{X} e_{Y}=\left(1-e_{X}\right) e_{Y}+e_{X \cap Y}$. Since $|X \cap Y|<|X|$, for each $X \in K$, we see that $e_{X \cap Y} \in N$, and so $e_{Y} \in N_{X}$.

Now, let $M_{X}$ be a maximal right ideal of $Q$ containing $N_{X}$. Since $e_{Y} \in N_{X} \subseteq$ $M_{X}$, we see that the simple right $Q$-module $Q / M_{X}$ is annihilated by $e_{Y}$, for each $Y \in K, Y \neq X$. It is clear, however, that $e_{X} \notin M_{X}$. Then we can define a set of simple right $Q$-modules indexed by $K$, by setting $C_{k}=Q / M_{k}$ for each $k \in K$, and we have that $C_{k} e_{k} \neq 0$ and $C_{j} e_{k}=0$ for each $k, j \in K, j \neq k$. Furthermore, since the $e_{k}$ are central idempotents for all $k \in K$, we have that $C_{j} \cong C_{k}$ implies $j=k$.

Let $P_{R}$ be a finitely generated projective right $R$-module, $E=E\left(P_{R}\right)$ its injective envelope, $S=\operatorname{End}\left(E_{R}\right)$ and $J=J(S)$. We want to use Lemma 2.3 applied to $S / J$ to deduce that $P_{R}$ has finite essential socle under suitable additional hypotheses. However, the situation here is different from the one considered in [14, Theorem 1], and this cannot be done directly. More precisely, we need to devise a way to pass 
injectively from (isomorphism classes of) simple right $S / J$-modules to simple right $R$-modules. The following lemma provides this injection and will play a crucial role in our main result.

Lemma 2.4. Let $P_{R}$ be a finitely generated projective module, $E=E\left(P_{R}\right), S=$ $\operatorname{End}_{R}(E)$, and $J=J(S)$. Assume that each cyclic submodule of $E_{R}$ is essentially embeddable in a projective module. Then, if $\left\{C_{k}\right\}_{k \in K}$ is an idempotent-orthogonal family of simple right $S / J$-modules, there exists an injective mapping from $K$ to the set of isomorphism classes of simple right $R$-modules.

Proof. Since idempotents of $S / J$ lift modulo $J$, we may consider a family $\left\{s_{k}\right\}_{k \in K}$ of idempotents of $S$ such that if $\bar{s}_{k}=s_{k}+J$ then $C_{k} \bar{s}_{k} \neq 0$, and $C_{k} \bar{s}_{j}=0$ for $k, j \in K, k \neq j$. It is then clear that $C_{k} s_{k} \neq 0$, and $C_{k} s_{j}=0$ for $k \neq j$. For each $k \in K$ let $E_{k}=I m s_{k}$, and $i_{k}: E_{k} \rightarrow E$ the canonical inclusion. Then $E_{k}$ is a direct summand of $E$ and, hence, an injective module. For each $k \in K$, let $c_{k} \in C_{k}$ be an element such that $c_{k} s_{k} \neq 0$, and call $p_{k}: S_{S} \rightarrow C_{k}$ the homomorphism of Mod- $S$ defined by $p_{k}(1)=c_{k}$. Then $p_{k}\left(s_{k}\right) \neq 0$, and we claim that also $\left(p_{k} \otimes_{S} E\right) \circ s_{k} \neq 0$.

To simplify the notation, denote by ()$_{*}$ the functor $\operatorname{Hom}_{R}(E,-)$, so that, in particular, $s_{k_{*}}=\operatorname{Hom}_{R}\left(E, s_{k}\right)$ is the endomorphism of $S_{S}$ given by left multiplication with $s_{k}$. Then $p_{k}\left(s_{k}\right)=\left(p_{k} \circ s_{k *}\right)(1)$, and we see that $p_{k} \circ s_{k *} \neq 0$, so that $g:=p_{k} \circ s_{k_{*}}: S \rightarrow C_{k}$ is an epimorphism. Since it is clear that $s_{k_{*}} \otimes_{S} E=$ $H_{o m}\left(E, s_{k}\right) \otimes_{S} E$ can be identified with $s_{k}$, in order to prove our claim it is enough to show that the morphism $g \otimes_{S} E=\left(p_{k} \otimes_{S} E\right) \circ\left(s_{k *} \otimes_{S} E\right): E \rightarrow E \otimes_{S} C_{k}$ is nonzero. Let $H=K e r g$. Then the short exact sequence of Mod-S, $0 \rightarrow H \longrightarrow$ $S \stackrel{g}{\longrightarrow} C_{k} \rightarrow 0$, induces a short exact sequence in Mod- $R$ :

$$
0 \rightarrow H E \longrightarrow E \stackrel{g \otimes_{S} E}{\longrightarrow} C_{k} \otimes_{S} E \rightarrow 0 .
$$

Assume that $H E=E$. Since $P$ is a finitely generated submodule of $E$, this implies that we can find an integer $r \geq 0$ and a homomorphism $f: E^{r} \rightarrow E$ such that $P \subseteq \operatorname{Im} f$ and if $q_{i}: E \rightarrow E^{r}, i=1, \ldots, r$, denote the injections, then $f \circ q_{i} \in H$ for all $i=1, \ldots, r$. Let $\gamma: P \rightarrow E$ be the canonical inclusion. Then, since $P$ is projective, there exists a homomorphism $\delta: P \rightarrow E^{r}$ such that $\gamma=f \circ \delta$. Furthermore, since $E^{r}$ is injective, we obtain $\eta: E \rightarrow E^{r}$ such that $\eta \circ \gamma=\delta$. Thus, in the diagram

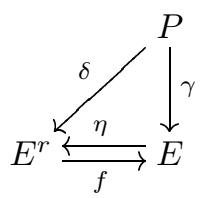

we have that $f \circ \eta \circ \gamma=f \circ \delta=\gamma$, and so $(1-f \circ \eta) \circ \gamma=0$. Since $P$ is essential in $E$, this implies that $\operatorname{Ker}(1-f \circ \eta)$ is essential in $E$ and hence that $1-f \circ \eta \in J$. Therefore, $f \circ \eta$ is an isomorphism. If we denote by $p_{i}: E^{r} \rightarrow E$ the canonical projections, we see that

$$
f \circ \eta=f \circ\left(\sum_{i=1}^{r} q_{i} \circ p_{i}\right) \circ \eta=\sum_{i=1}^{r}\left(\left(f \circ q_{i}\right) \circ\left(p_{i} \circ \eta\right)\right) \in H .
$$

Hence we must have that $H=S$, a contradiction that proves our claim. Since, for each $k \in K,\left(p_{k} \otimes_{S} E\right) \circ s_{k} \neq 0$, we also have that $\left(p_{k} \otimes_{S} E\right) \circ i_{k} \neq 0$, and so there exists an element $x_{k} \in E_{k}$ such that $\left(\left(p_{k} \otimes_{S} E\right) \circ i_{k}\right)\left(x_{k}\right) \neq 0$. Since the cyclic right $R$-module $x_{k} R$ is a submodule of $E$, our hypothesis implies that there exist 
a projective right $R$-module $P_{k}$ and an essential monomorphism $v_{k}: x_{k} R \rightarrow P_{k}$. Then, if $u_{k}$ denotes the canonical inclusion of $x_{k} R$ in $E_{k}$, we have by injectivity a homomorphism $h_{k}: P_{k} \rightarrow E_{k}$ such that $h_{k} \circ v_{k}=u_{k}$. Furthermore, as $v_{k}$ is essential, $h_{k}$ is a monomorphism.

Now, let $t_{k}:=i_{k} \circ h_{k}$. Then $t_{k} \circ v_{k}=i_{k} \circ h_{k} \circ v_{k}=i_{k} \circ u_{k}$, and thus we see that $\left(\left(p_{k} \otimes_{S} E\right) \circ t_{k}\right)\left(v_{k}\left(x_{k}\right)\right)=\left(\left(p_{k} \otimes_{S} E\right) \circ i_{k} \circ u_{k}\right)\left(x_{k}\right) \neq 0$, so that $\left(p_{k} \otimes_{S} E\right) \circ t_{k} \neq 0$. Thus this morphism has nonzero image, say $N_{k}=\operatorname{Im}\left(\left(p_{k} \otimes_{S} E\right) \circ t_{k}\right)$, with canonical projection $q_{k}: P_{k} \rightarrow N_{k}$ and inclusion $w_{k}: N_{k} \rightarrow C_{k} \otimes_{S} E$.

We claim that $N_{k}$ is, actually, a finitely generated module. To see this, observe that $P_{k}$ is a direct summand of a free right $R$-module, say $R^{(I)}$ with inclusion $\varepsilon: P_{k} \rightarrow R^{(I)}$ and projection $\theta: R^{(I)} \rightarrow P_{k}$ satisfying $\theta \circ \varepsilon=1_{P_{k}}$. Thus we have a monomorphism $\varepsilon \circ v_{k}: x_{k} R \rightarrow R^{(I)}$, and so there exists a finite subset $I_{0} \subseteq I$ such that $\operatorname{Im}\left(\varepsilon \circ v_{k}\right) \subseteq R^{\left(I_{0}\right)}$. Let $\iota_{0}: R^{\left(I_{0}\right)} \rightarrow R^{(I)}$ be the inclusion and $\pi_{0}: R^{(I)} \rightarrow R^{\left(I_{0}\right)}$ the projection. Then we have

$$
v_{k}=\theta \circ \varepsilon \circ v_{k}=\theta \circ \iota_{0} \circ \pi_{0} \circ \varepsilon \circ v_{k}=\lambda_{k} \circ v_{k},
$$

where $\lambda_{k}:=\theta \circ \iota_{0} \circ \pi_{0} \circ \varepsilon$. Let $E_{k}^{\prime}=E\left(x_{k} R\right) \subseteq E_{k}, i_{k}^{\prime}: E_{k}^{\prime} \rightarrow E$ the inclusion and $h_{k}^{\prime}: P_{k} \rightarrow E_{k}^{\prime}$ the morphism induced by $h_{k}$, so that $t_{k}=i_{k}^{\prime} \circ h_{k}^{\prime}$. By injectivity of $E$ we obtain a homomorphism $i_{k}^{\prime \prime}: E_{k}^{\prime} \rightarrow E$ making the following diagram commute:

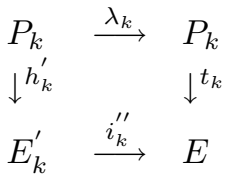

and so we have

$$
i_{k}^{\prime \prime} \circ h_{k}^{\prime} \circ v_{k}=t_{k} \circ \lambda_{k} \circ v_{k}=t_{k} \circ v_{k}=i_{k}^{\prime} \circ h_{k}^{\prime} \circ v_{k} .
$$

Thus we see that $x_{k} R \subseteq \operatorname{Ker}\left(i_{k}^{\prime}-i_{k}^{\prime \prime}\right)$, and hence that $\operatorname{Ker}\left(i_{k}^{\prime}-i_{k}^{\prime \prime}\right)$ is essential in $E_{k}^{\prime}$. Since $E_{k}^{\prime}$ is a direct summand of $E$, we can extend $i_{k}^{\prime}-i_{k}^{\prime \prime}$ to an endomorphism $z_{k}$ of $E$ with essential kernel, so that $z_{k} \in J(S)$ and $\left.z_{k}\right|_{E_{k}^{\prime}}=i_{k}^{\prime}-i_{k}^{\prime \prime}$. We have that

$$
\left(p_{k} \otimes E\right) \circ z_{k}=\left(p_{k} \otimes E\right) \circ\left(z_{k *} \otimes E\right)=\left(p_{k} \circ z_{k *}\right) \otimes E,
$$

where $z_{k *}=\operatorname{Hom}_{R}\left(E, z_{k}\right): S_{S} \rightarrow S_{S}$ is given by $z_{k *}(1)=z_{k}$, and so $\left(p_{k} \circ z_{k *}\right)(1)=$ $p_{k}\left(z_{k}\right)=0$ (since $z_{k} \in J(S)$ ). Thus we see that $\left(p_{k} \otimes E\right) \circ z_{k}=0$, and hence that $\left(p_{k} \otimes E\right) \circ i_{k}^{\prime}=\left(p_{k} \otimes E\right) \circ i_{k}^{\prime \prime}$ and $\left(p_{k} \otimes E\right) \circ t_{k}=\left(p_{k} \otimes E\right) \circ t_{k} \circ \lambda_{k}$. Observe now that

$$
\begin{aligned}
N_{k}=\operatorname{Im}\left(\left(p_{k} \otimes E\right) \circ t_{k} \circ \lambda_{k}\right) & \subseteq \operatorname{Im}\left(\left(p_{k} \otimes E\right) \circ t_{k} \circ \theta \circ \iota_{0}\right) \\
& \subseteq \operatorname{Im}\left(\left(p_{k} \otimes E\right) \circ t_{k}\right)=N_{k} .
\end{aligned}
$$

Thus $N_{k}=\operatorname{Im}\left(\left(p_{k} \otimes E\right) \circ t_{k} \circ \theta \circ \iota_{0}\right)$, where

$$
\left(p_{k} \otimes E\right) \circ t_{k} \circ \theta \circ \iota_{0}: R^{\left(I_{0}\right)} \rightarrow C_{k} \otimes E
$$

has finitely generated domain. This shows that $N_{k}$ is indeed finitely generated.

Since $N_{k}$ is finitely generated we can choose, for each $k \in K$, a simple quotient $U_{k}$ of $N_{k}$ with canonical projection $\pi_{k}: N_{k} \rightarrow U_{k}$. Denoting by $[X]$ the isomorphism class of a right $R$-module $X$, we define a map from $K$ to the set of isomorphism classes of simple right $R$-modules by assigning $k \mapsto\left[U_{k}\right]$.

To complete the proof of the lemma, we show that the map just defined is injective. So, assume that $\left[U_{j}\right]=\left[U_{k}\right]$ for $j, k \in K$, and let $\varphi: U_{j} \rightarrow U_{k}$ be an 
isomorphism. If $\alpha_{k}: U_{k} \rightarrow E\left(U_{k}\right)$ denotes the inclusion, for each $k \in K$, we obtain by injectivity an $R$-homomorphism $\phi: E\left(U_{j}\right) \rightarrow E\left(U_{k}\right)$ satisfying $\phi \circ \alpha_{j}=\alpha_{k} \circ \varphi$. Also, $\alpha_{k} \circ \pi_{k}$ has an extension $\pi_{k}^{\prime}$ to $C_{k} \otimes_{S} E$, so $\alpha_{k} \circ \pi_{k}=\pi_{k}^{\prime} \circ w_{k}$. On the other hand, using the projectivity of $P_{j}$, we obtain a homomorphism $\psi: P_{j} \rightarrow P_{k}$ such that $\pi_{k} \circ q_{k} \circ \psi=\varphi \circ \pi_{j} \circ q_{j}$. Now, from the injectivity of $E$, we obtain an endomorphism $\tau: E \rightarrow E$, i.e., an element $\tau \in S$, such that $\tau \circ t_{j}=t_{k} \circ \psi$. Observe then that $\phi \circ \alpha_{j}=\alpha_{k} \circ \varphi$ is a monomorphism, and hence the morphism $\phi \circ \alpha_{j} \circ \pi_{j} \circ q_{j}: P_{j} \rightarrow E\left(U_{k}\right)$ is nonzero (with image isomorphic to $U_{j}$ ). We have a commutative diagram

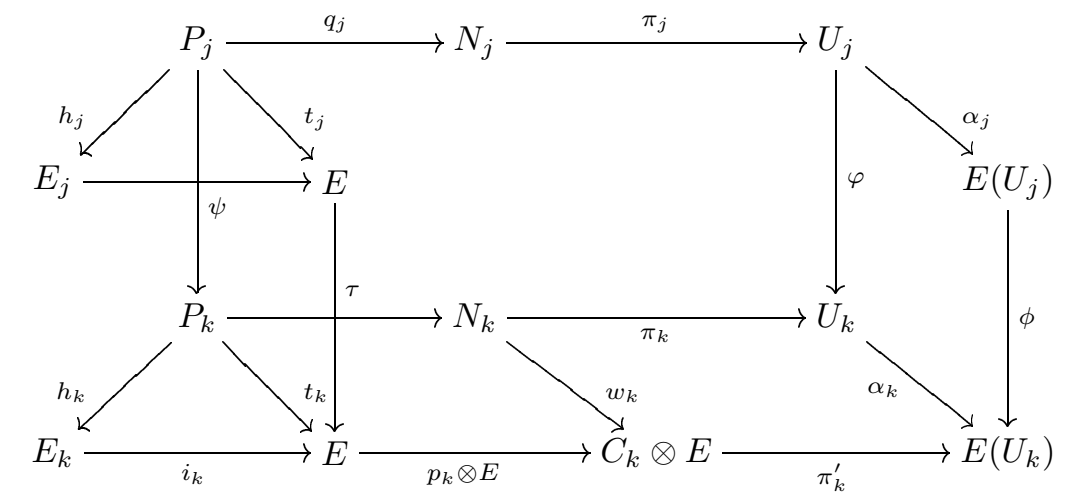

and so we see that

$$
\begin{aligned}
0 & \neq \phi \circ \alpha_{j} \circ \pi_{j} \circ q_{j}=\alpha_{k} \circ \varphi \circ \pi_{j} \circ q_{j}=\alpha_{k} \circ \pi_{k} \circ q_{k} \circ \psi=\pi_{k}^{\prime} \circ w_{k} \circ q_{k} \circ \psi \\
& =\pi_{k}^{\prime} \circ\left(p_{k} \otimes_{S} E\right) \circ t_{k} \circ \psi=\pi_{k}^{\prime} \circ\left(p_{k} \otimes_{S} E\right) \circ \tau \circ t_{j} \\
& =\pi_{k}^{\prime} \circ\left(p_{k} \otimes_{S} E\right) \circ \tau \circ i_{j} \circ h_{j} .
\end{aligned}
$$

Assume now that $j \neq k$ and consider the homomorphism $p_{k} \circ \tau_{*} \circ i_{j_{*}}: s_{j} S \rightarrow C_{k}$, where $\tau_{*}=\operatorname{Hom}_{R}(E, \tau)$ and $i_{j_{*}}=\operatorname{Hom}_{R}\left(E, i_{j}\right)$. If we set $x:=\left(p_{k} \circ \tau_{*}\right)(1) \in C_{k}$, we have that $\left(p_{k} \circ \tau_{*} \circ i_{j_{*}}\right)\left(s_{j}\right)=\left(p_{k} \circ \tau_{*}\right)\left(s_{j}\right)=x s_{j} \in C_{k} s_{j}=0$. Tensoring with ${ }_{S} E$, we then see that $\left(p_{k} \otimes_{S} E\right) \circ\left(\tau_{*} \otimes_{S} E\right) \circ\left(i_{j_{*}} \otimes_{S} E\right)=0$ and, since $\tau_{*} \otimes_{S} E \cong \tau$ and $i_{j_{*}} \otimes_{S} E \cong i_{j}$, that $\left(p_{k} \otimes_{S} E\right) \circ \tau \circ i_{j}=0$, which gives a contradiction and shows that we must have $j=k$.

\section{Main Results}

We are now ready to give our main result. We will use the following notation (cf. [13]). If $R$ is a ring, $\Omega(R)$ will denote a set of representatives of the isomorphism classes of simple right $R$-modules. If $M$ is a right $R$-module, then $C(M)$ denotes a set of representatives of the isomorphism classes of simple submodules of $M$.

Theorem 3.1. Let $R$ be a ring and $P_{R}$ a finitely generated projective module. Assume that $|\Omega(R)| \leq|C(P)|$ and that every cyclic submodule of $E\left(P_{R}\right)$ is essentially embeddable in a projective module. Then $P_{R}$ cogenerates the simple right $R$-modules and has finite essential socle.

Proof. Let $E_{R}=E\left(P_{R}\right), S=E n d\left(E_{R}\right)$, and $J=J(S)$. By Lemma 2.1, there exists a bijection between the set $\mathcal{E}$ of isomorphism classes of indecomposable direct summands of $E_{R}$ and the set $\mathcal{M}$ of isomorphism classes of minimal right ideals of 
$S / J$. It is clear that the assignment $[C] \mapsto[E(C)]$ defines an injection from $C(P)$ to $\mathcal{E}$, and so we have that $|\Omega(R)|=|C(P)| \leq|\mathcal{E}|=|\mathcal{M}|$. Assume that $|\Omega(R)|$ is infinite. Then $|\mathcal{M}|$ is also infinite and, by Lemma 2.3 , there exists an idempotentorthogonal family $\left\{C_{k}\right\}_{k \in K}$ of simple right $S / J$-modules, such that $|\mathcal{M}|<|K|$. By Lemma 2.4, there is an injective mapping from $K$ to $\Omega(R)$, and so we get a chain of inequalities:

$$
|\Omega(R)| \leq|\mathcal{M}|<|K| \leq|\Omega(R)|,
$$

which gives a contradiction and proves that $|\Omega(R)|$ is finite. Observe also that, since $|\Omega(R)|=|C(P)|$ is finite and $C(P) \subseteq \Omega(R)$, we have that, actually, $\Omega(R)=C(P)$. This means that $P_{R}$ cogenerates all the simple right $R$-modules.

Let $|\Omega(R)|=n$. We claim that $|\mathcal{M}|=n$ too. Let $C_{1}, \ldots, C_{r}$ be a set of representatives of the elements of $\mathcal{M}$. We already know that $n \leq r$. Furthermore, there exist idempotent elements $e_{1}, e_{2}, \ldots, e_{r} \in S$ such that, if $\bar{e}_{i}=e_{i}+J$ for $i=1, \ldots, r$, then $C_{i}=\bar{e}_{i}(S / J)$. Since $\bar{e}_{i}(S / J) \bar{e}_{j}=\operatorname{Hom}_{S / J}\left(\bar{e}_{j}(S / J), \bar{e}_{i}(S / J)\right)$, we see that $\bar{e}_{i}(S / J) \bar{e}_{j}=0$ for $i \neq j$ and $\bar{e}_{i}(S / J) \bar{e}_{i} \neq 0$ for all $i=1, \ldots, r$. Thus the family $\left\{C_{i}\right\}_{i=1, \ldots, r}$ is an idempotent-orthogonal family of simple right $S / J$ modules with respect to the idempotents $\left\{\bar{e}_{i}\right\}_{i=1, \ldots, r}$. From Lemma 2.4 we obtain that $r \leq|\Omega(R)|=n$, and so we have $n=r$.

Now, we claim that $S / J$ is a semisimple ring. Assume that this is not the case. Then there exists a nonprojective simple right $S / J$-module $C$ which is not isomorphic to any of the $C_{i}=\bar{e}_{i}(S / J), i=1, \ldots, n$. First, we show that $C e_{i}=0$ for each $i=1, \ldots, n$. Assume, on the contrary, that there exists an element $c \in C$ such that $c e_{i} \neq 0$ for some $i \in\{1, \ldots, n\}$. Then $c \bar{e}_{i} \neq 0$ and $\left(c \bar{e}_{i}\right)(S / J)$ is a nonzero right $S / J$-submodule of $C$, so that $\left(c \bar{e}_{i}\right)(S / J)=C$. Consider now the homomorphism of right $S / J$-modules from $C_{i}$ to $C$ given by left multiplication with $c$. It is a nonzero homomorphism between two simple right $S / J$-modules, and so we see that $C_{i} \cong C$, a contradiction. Thus we have established that $C e_{i}=0$ for all $i=1, \ldots, n$.

Next let $\left[U_{1}\right], \ldots,\left[U_{n}\right]$ be the isomorphism classes of right $R$-modules corresponding to the simple $S / J$-modules $C_{1}, \ldots, C_{n}$ in an injective mapping obtained from Lemma 2.4. We are going to show that we can assign, in a similar way, a simple right $R$-module $U$ to $C$, with the property that $U$ is not isomorphic to any of the $U_{i}$, for $i=1, \ldots, n$. Let $0 \neq x \in C$, and let $p: S_{S} \rightarrow C$ be the projection defined by $p(1)=x$. Then the argument used in the first part of the proof of Lemma 2.4 shows that $p \otimes_{S} E: E \rightarrow C \otimes_{S} E$ is a nonzero homomorphism. Let $X$ be a nonzero finitely generated submodule of $E$ which is not contained in $\operatorname{Ker}\left(p \otimes_{S} E\right)$, and set $N=\left(p \otimes_{S} E\right)(X)$, with projection $q: X \rightarrow N$. Let $U$ be a simple quotient of the finitely generated right $R$-module $N$, with canonical projection $\pi: N \rightarrow U$.

Let $j \in\{1, \ldots, n\}$, and $\varphi: U_{j} \rightarrow U$ an isomorphism of right $R$-modules. We obtain as in the proof of Lemma 2.4 a homomorphism $\phi: E\left(U_{j}\right) \rightarrow E(U)$ satisfying $\phi \circ \alpha_{j}=\alpha \circ \varphi$, where $\alpha_{j}: U_{j} \rightarrow E\left(U_{j}\right)$ and $\alpha: U \rightarrow E(U)$ are the inclusions. Similarly, keeping the notation of Lemma 2.4 , we have a homomorphism $\psi: P_{j} \rightarrow X$ (obtained from the projectivity of $P_{j}$ ) that satisfies $\varphi \circ \pi_{j} \circ q_{j}=\pi \circ q \circ \psi$. Let $t: X \rightarrow E$ and $w: N \rightarrow C \otimes_{S} E$ be the inclusions. By the injectivity of $E(U)$ there is a homomorphism $\pi^{\prime}: C \otimes_{S} E \rightarrow E(U)$ such that $\pi^{\prime} \circ w=\alpha \circ \pi$. As in Lemma 2.4 we have the inclusion $i_{j}: E_{j}=e_{j} E \rightarrow E$, and morphisms $h_{j}: P_{j} \rightarrow E_{j}$ and $t_{j}: P \rightarrow E$, with $t_{j}=i_{j} \circ h_{j}$. Similarly, we have an endomorphism $\tau: E \rightarrow E$ such that $\tau \circ t_{j}=t \circ \psi$. 
Then we have

$$
\begin{aligned}
0 & \neq \phi \circ \alpha_{j} \circ \pi_{j} \circ q_{j}=\alpha \circ \varphi \circ \pi_{j} \circ q_{j}=\alpha \circ \pi \circ q \circ \psi=\pi^{\prime} \circ w \circ q \circ \psi \\
& =\pi^{\prime} \circ\left(p \otimes_{S} E\right) \circ t \circ \psi=\pi^{\prime} \circ\left(p \otimes_{S} E\right) \circ \tau \circ t_{j} \\
& =\pi^{\prime} \circ\left(p \otimes_{S} E\right) \circ \tau \circ i_{j} \circ h_{j} .
\end{aligned}
$$

Exactly as in Lemma 2.4, we consider the homomorphism $p \circ \tau_{*} \circ i_{j_{*}}: e_{j} S \rightarrow C$, where $\tau_{*}=H_{0 m_{R}}(E, \tau)$ and $i_{j_{*}}=H_{o m}\left(E, i_{j}\right)$. We obtain that $\left(p \otimes_{S} E\right) \circ \tau \circ i_{j}=$ 0 , which gives a contradiction and shows that $S / J$ is semisimple. Therefore, $S$ is a semiperfect ring and $E_{R}$ is a finite-dimensional module. Thus $E_{R}$ is a finite direct sum of indecomposable submodules. Since $|\mathcal{E}| \leq|\mathcal{M}|=n$, we see that each of these indecomposables is, actually, an injective envelope of a simple right $R$-module, so that $E_{R}$, and hence $P_{R}$, has finite essential socle.

In [13], Menal adapted Osofsky's counting argument in order to prove that if $R$ is a ring and $P_{R}$ is a finitely generated projective and injective module such that $|\Omega(R)| \leq|C(P)|$, then $|\Omega(R)|$ is finite $([13$, Theorem 8]). We see that this is a particular case of Theorem 3.1. Menal also proved ([13, Corollary 8$])$ that the rings $R$ such that every cyclic right $R$-module embeds in a free module and the injective envelope $E\left(R_{R}\right)$ is projective, are QF (cf. [13, Corollary 9]). We have the following more general result.

Corollary 3.2. Let $R$ be a ring such that $E\left(R_{R}\right)$ is a projective cogenerator of Mod- $R$. Then $R$ is right $P F$.

Proof. It is clear that the hypotheses of Theorem 3.1 are satisfied, and we conclude that $R$ has finitely generated essential socle. Let $C_{1}, \ldots, C_{n}$ be a set of representatives of the isomorphism classes of simple right $R$-modules. Their injective envelopes are indecomposable, projective and injective. Thus, if $J=J(R)$, we have that the modules $T_{i}:=E\left(C_{i}\right) / E\left(C_{i}\right) J$ are pairwise nonisomorphic simple right $R$-modules. Therefore, the $T_{i}$ are also a set of representatives of the isomorphism classes of simple right $R$-modules, and, since all of them have a projective cover (the $E\left(C_{i}\right)$ ), we see that $R$ is semiperfect. Then $R_{R}$ has a direct decomposition $R=\bigoplus_{i=1}^{r} e_{i} R$, where the $e_{i} R$ are all projective covers of simple modules, so that each of them is isomorphic to one of the $E\left(C_{i}\right)$. Thus we see that $R$ is right self-injective and, therefore, right $\mathrm{PF}$.

Recall from [1] that a ring $R$ is called right CES when every cyclic right $R$ module is essentially embeddable in a direct summand of $R_{R}$, and, more generally, $R$ is right CEP $[1,10]$ when every cyclic right $R$-module is essentially embeddable in a projective module. Semiperfect right CEP rings were studied in $[1,10,11]$. In [1], the question is posed of whether a right CEP ring or a right CES ring is already semiperfect, a fact that is proved in that paper assuming some additional conditions on $R$. The following corollary provides a general affirmative answer to this question.

Corollary 3.3. Every right CEP ring is right artinian. In particular, every right CES ring is right artinian.

Proof. From Theorem 3.1 we know that $R_{R}$ has finite essential socle. Since every cyclic right $R$-module embeds in a finitely generated free module, it has also finite essential socle. It is well known that this implies that $R$ is right artinian. 
It follows from the preceding corollary that the results obtained in $[1,10,9,11]$ for semiperfect right CEP or right CES rings hold for all right CEP and right CES rings. We mention, in particular, that in [9], a structure theorem is given for semiperfect right CES rings. We can now lift the semiperfect condition and observe that Corollary 3.3 together with [9, Theorem 3.5] or [1, Theorem B] gives the following structure result.

Corollary 3.4. Let $R$ be a ring. Then, the following conditions are equivalent:

(i) $R$ is right $C E S$.

(ii) $R$ is of one of the following types:

(a) $R$ is (artinian) uniserial as a right $R$-module,

(b) $R$ is an $n \times n$ matrix ring over a right self-injective ring of type (a), or

(c) $R$ is a direct sum of rings of types (a) or (b).

The rings that satisfy the hypotheses of Theorem 3.1 , with $P=R$, need not be right $\mathrm{PF}$, even when they are right artinian (as is shown by the examples of right CEP rings given in [10]). However, as it was pointed out in the introduction, the Levy-Faith problem of whether a right FGF ring is necessarily QF is still open (see [6], where several conditions are given that force a right FGF ring to be $\mathrm{QF}$ ). The following corollary gives a partial positive answer to this problem.

Corollary 3.5. Let $R$ be a ring such that every finitely generated right $R$-module is essentially embeddable in a projective module. Then $R$ is a QF ring.

Proof. By Theorem 3.1, $R$ is right artinian, so that $R$ is a QF ring by [6, Theorem $3.2]$.

Recall that a ring $R$ is called right CS when every complement right ideal is a direct summand of $R_{R}$ or, equivalently, every right ideal is essential in a direct summand of $R_{R}$ (cf. [3]). CS rings and CS modules (defined in a similar way and called also extending modules) have been object of intensive study in recent years (cf. [4]). Our methods allow us to prove that the CS condition is strong enough to force a right FGF ring to become QF. In order to do that, we will use the following auxiliary result that has been proved in [8, Lemma 1.5].

Lemma 3.6. Let $P_{R}$ be a finitely generated projective right $R$-module, $E=E\left(P_{R}\right)$ its injective hull, and $S=\operatorname{End}\left(E_{R}\right)$. Assume that each finitely generated submodule of $E$ embeds in a finitely presented module of projective dimension $\leq 1$. Then, for each finitely generated right $S / J$-module $X, \operatorname{Hom}_{R}\left(E / P, X \otimes_{S} E\right)=0$.

Theorem 3.7. Let $R$ be a right FGF right CS ring. Then $R$ is $Q F$.

Proof. By [6], it is enough to show that $R_{R}$ has finite essential socle. To prove this, observe first that we can modify the proof of Lemma 2.4 to show that the assertion of this lemma also holds in our current hypotheses. Indeed, let $E=E\left(R_{R}\right), S=$ $\operatorname{End}\left(R_{R}\right)$, and $J=J(S)$, and consider an idempotent-orthogonal family $\left\{C_{k}\right\}_{k \in K}$ of simple right $S / J$-modules, with respect to a family $\left\{s_{k}\right\}$ of idempotents of $S$. As in the proof of Lemma 2.4, we have that if $E_{k}=s_{k} E$ and $i_{k}: E_{k} \rightarrow E$ is the inclusion, for each $k \in K$, then $\left(p_{k} \otimes_{S} E\right) \circ s_{k} \neq 0$ and hence $\left(p_{k} \otimes_{S} E\right) \circ i_{k} \neq 0$. Set $L_{k}:=E_{k} \cap R$. Since $L_{k}$ is essential in $E_{k}$, we have that $E_{k}=E\left(L_{k}\right)$. On the other hand, since $R$ is right CS, there exist a direct summand $P_{k}=e_{k} R$ of $R_{R}$ (with $e_{k} \in S$ an idempotent) and an essential monomorphism $v_{k}: L_{k} \rightarrow P_{k}$. 
Then, $P_{k}$ embeds in $E_{k}$ and, in fact, since this embedding is essential, we see that $E_{k}=E\left(P_{k}\right)$ is an injective envelope of $P_{k}$.

Let $h_{k}: P_{k} \rightarrow E_{k}$ be the inclusion and $t_{k}:=i_{k} \circ h_{k}$. Assume $\left(p_{k} \otimes_{S} E\right)$ $\circ t_{k}=0$. Then $\left(p_{k} \otimes_{S} E\right) \circ i_{k}$ factors through $E_{k} / P_{k}$. On the other hand, if $P_{k}^{\prime}:=\left(1-e_{k}\right) R$ and $E_{k}^{\prime}:=E\left(P_{k}^{\prime}\right)$, it is clear that $E \cong E_{k} \oplus E_{k}^{\prime}$, and so $E / R \cong$ $\left(E_{k} / P_{k}\right) \oplus\left(E_{k}^{\prime} / P_{k}^{\prime}\right)$. Since $s_{k} E_{k}^{\prime}=0$, we see that $\left(p_{k} \otimes_{S} E\right) \circ s_{k}$ factors through $E / R$. Then it follows from Lemma 3.6 that $\left(p_{k} \otimes_{S} E\right) \circ s_{k}=0$, which is a contradiction and hence shows that $\left(p_{k} \otimes_{S} E\right) \circ t_{k} \neq 0$.

From this point on, we can follow the proof of Lemma 2.4 to conclude that, again, there is an injective mapping from $K$ to the set of isomorphism classes of simple right $R$-modules. Observe now that in the proof of Theorem 3.1, the hypothesis of the essential embedding of the cyclic submodules of $E$ in projectives is only used in order to let us apply Lemma 2.4. Thus we see that the arguments used in that proof carry over to this situation, and we conclude that $R_{R}$ has finite essential socle, completing the proof.

\section{REFERENCES}

[1] A. Al-Huzali, S.K. Jain and S.R. López-Permouth, On the weak relative-injectivity of rings and modules, Lecture Notes in Math., vol. 1448, Springer-Verlag, Berlin and New York, 1989, pp. 93-98. MR 92d:16006

[2] J.E. Björk, Radical properties of perfect modules, J. Reine Angew. Math. 245 (1972), 78-86. MR 47:1864

[3] A.W. Chatters and C.R. Hajarnavis, Rings in which every complement right ideal is a direct summand, Quart. J. Math. Oxford Ser. (2) 28 (1977), 61-80. MR 55:10519

[4] Nguyen Viet Dung, Dinh Van Huynh, P. Smith, and R. Wisbauer, Extending modules, Pitman Res. Notes in Math., vol. 313, Longman, Harlow, 1994. MR 96f: 16008

[5] C. Faith, Algebra. II: Ring Theory, Springer-Verlag, Berlin and New York, 1976. MR 55:383

[6] C. Faith, Embedding modules in projectives. A report on a problem, Lecture Notes in Math., vol. 951, Springer-Verlag, Berlin and New York, 1982, pp. 21-40. MR 84i:16001

[7] C. Faith, Embedding torsionless modules in projectives, Publ. Mat. 34 (1990), 379-387. MR 92b:16016

[8] J.L. Gómez Pardo and P.A. Guil Asensio, Endomorphism rings of completely pure-injective modules, Proc. Amer. Math. Soc. 124 (1996), 2301-2309. MR 96j:16029

[9] S.K. Jain and S.R. López-Permouth, A generalization of the Wedderburn-Artin theorem, Proc. Amer. Math. Soc. 106 (1989), 19-23. MR 89i:16013

[10] S.K. Jain and S.R. López-Permouth, Rings whose cyclics are essentially embeddable in projective modules, J. Algebra 128 (1990), 257-269. MR 90k:16016

[11] S.K. Jain, S.R. López-Permouth and S. Singh, On a class of QI-rings, Glasgow Math. J. 34 (1992), 75-81. MR 93e: 16008

[12] L.S. Levy, Torsion-free and divisible modules over non-integral domains, Canad. J. Math. 15 (1963), 132-151. MR 26:155

[13] P. Menal, On the endomorphism ring of a free module, Publ. Mat. Univ. Autonoma Barcelona 27 (1983), 141-154. MR 86g:16046

[14] B.L. Osofsky, A generalization of quasi-Frobenius rings, J. Algebra 4 (1966), 373-387; errata, 9 (1968), 120. MR 34:4305; MR 36:6443 SPAIN

Departamento de Algebra, Universidad de Santiago, 15771 Santiago de Compostela,

E-mail address: pardo@zmat.usc.es

Departamento de Matematicas, Universidad de Murcia, 30100 Espinardo, Murcia, SPAIN

E-mail address: paguil@fcu.um.es 